\title{
Die frühe Sportpolitik des portugiesischen Estado Novo im Spiegel der Vereinspresse
}

\author{
Club newspapers in Lisbon and the early sports politics \\ of the Portuguese Estado Novo \\ A antiga política desportiva do Estado Novo português \\ no espelho da imprensa do clube
}

Thomas Weißmann

Technische Universität Chemnitz, Chemnitz / Alemanha Mestre em História, Universität Kassel thomas.weissmann@phil.tu-chemnitz.de

\begin{abstract}
RESumo: 0 artigo debruçar-se-á sobre a maneira da imprensa clubista lisboeta de responder à implementação do desporto português independente na orgânica do Estado Novo por decretos no início dos anos 1940. Seguindo a argumentação das entidades oficiais que os clubes têm de contribuir para a educação física do povo português, a imprensa clubista conseguiu tornar aquele argumento de origem restritiva em favor dos clubes. Argumentando que os clubes possam melhor contribuir enquanto são menos taxados, a imprensa clubista usava também uma narrativa nacionalista que apoiou uma eficaz amplificação das margens da política clubista nos limites da ditadura.
\end{abstract}

PalaVRas-ChaVe: Ditadura; Portugal; Esportes.

ABSTRACT: The article aims to analyse how the publications of several Lisbon sports clubs responded to an order by several decrees to align the formerly independent sports structures with the regulatory and ideological framework of the Portuguese dictatorship in the early 1940s. While abiding to state requirements demanding that sports clubs cooperate in the education of the Portuguese population, the newspapers implemented a strategy which turned these originally restricting demands to their favour. Arguing that the clubs could better contribute to society with less taxation, and integrating such arguments in a nationalistic narrative successfully helped the clubs gain more political leeway in the late 1940s and in the 1950s.

KEYWORDS: Dictatorship; Portugal; Sports. 


\section{EINLEITUNG}

In Portugal stellte der Estado Novo mit dem Dekret 32241 vom 5. September 1942 die Sportvereine unter die staatliche Kontrolle der neugeschaffenen DirecçãoGeral da Educação Física, Desportos e Saúde Escolar (DGD). ${ }^{1}$ Die daraus resultierenden Aufgaben und Pflichten des Vereins- und Verbandssportes konkretisierte das Dekret 32946 vom 3. August 1943, in dem es unter anderem heißt, dass sich die Bevölkerung nur unzureichend durch staatliche Angebote zu Gymnastik und Sport verleiten lasse. ${ }^{2}$ Die sich bereits vor dem Militärputsch 1926 immer weiter ausdehnenden vereins- und verbandssportlichen Strukturen sollten folglich dabei mithelfen, ideologische Zielsetzungen $\mathrm{zu}$ erreichen. Ein Hauptaugenmerk des staatlichen Interesses lag auf der Gymnastik, die bereits in den zuvor geschaffenen Institutionen wie der Fundação Nacional para a Alegria no Trabalho (FNAT) oder der Mocidade Portuguesa (MP) betrieben wurde. Ziel war hierbei die Schaffung eines „Neuen Menschen“3 und die damit verbundene Überwindung der sogenannten Dekadenz des einst stolzen, aber nun degenerierten und rückständigen portugiesischen Volkes. ${ }^{4}$

Sport und Gymnastik fügen sich demnach in eine staatliche Kulturpolitik ein, die sich aus verschiedenen ideologischen Quellen speist. Folgt man der Argumentation Daniel Melos, handelt es sich um traditionelle Ansätze wie die Rückbesinnung auf den ruralen Charakter Portugals, aber auch um modernistische Ansätze, wobei vor allem die Orientierung an faschistische Modelle in den dreißiger und vierziger Jahren zu erkennen ist. ${ }^{5}$

Während der moderne Sport und insbesondere der Fußball für eine Vereinnahmung im Rahmen von Massenveranstaltungen geradezu prädestiniert ist, trägt er gleichzeitig soziale Sprengkraft für die Diktatur in sich, da er als englischer Import und auf Grund seiner immer stärkeren Ausbreitung in den

\footnotetext{
${ }_{1}^{1}$ MINISTÉRIO DA EDUCAÇÃO NACIONAL. Decreto-lei n. ${ }^{\circ}$ 32:241, S. 1135-1139.

${ }^{2}$ MINISTÉRIO DA EDUCAÇÃO NACIONAL. Decreto-lei n. ${ }^{\circ}$ 32:946. S. 491.

${ }^{3}$ ROSAS. O salazarismo e o homem novo: ensaio sobre o Estado Novo e a questão do totalitarismo, S. 1031-1054.

${ }_{5}^{4}$ KUMAR. A pureza perdida do desporto. Futebol no Estado Novo, S. 95-99.

${ }^{5}$ MELO. A cultura popular no Estado Novo, S. 14.
} 
Großstädten eine Kulturpolitik, die sich auf Tradition und genuin portugiesische Werte beruft, auf den ersten Blick eher konterkariert, denn fördert.

Es verwundert also nicht, dass den Vereinen durch die oben genannten Dekrete allerlei Restriktionen auferlegt worden sind, die in einem ersten Kapitel kurz erläutert werden. Anschließend folgt eine Analyse von Vereinszeitungen aus dem Lissaboner Großraum. ${ }^{6}$ Die dort geführten Diskussionen über die staatlichen Zielsetzungen im Bereich Sport lassen Strategien der Vereine erkennen, wie sie die Interessen des Staates und der Nation mit jenen der Vereine in Beziehung setzten und es dabei schafften, ursprünglich restriktive Argumente für die Vereinspolitik nutzbar zu machen. Dabei griff die Presseberichterstattung auch immer wieder auf nationalistische Narrative zurück und lässt sich im Bereich der inneren Politisierung7 des Sportes verorten, da die Vereine die vorgegebenen ideologischen Begrifflichkeiten einerseits mit Leben füllten und es ihnen andererseits Schritt für Schritt gelang, die Grenzen des Machbaren auch mit Hilfe der Vereinszeitungen langsam zu ihren Gunsten zu verschieben.

\section{DAS DEKRET 32946: VON STAATLICHER EINBETTUNG HIN ZU FORTSCHREITENDER EMANZIPIERUNG DES VERBANDSSPORTES}

Als der Staat in den vierziger Jahren den Vereinssport ideologisch und institutionell in seine Strukturen einbette, ist das Misstrauen gegenüber diesem neuartigen Phänomen immer noch deutlich spürbar. Wurden im Dekret 21110 vom 4. April

\footnotetext{
${ }^{6}$ Den Quellenkorpus bilden hierbei die Zeitungen der Vereine Sport Lisboa e Benfica, Sporting Clube de Portugal, Clube de Futebol "Os Belenenses" und Atlético Clube de Portugal, die in Auflage und Erscheinungszeiten variierten und bisweilen ihre Namen wechselten. Um den Fußnotenapparat nicht übermäßig aufzublähen, wird in der Regel indirekt zitiert und nur eine kleine Auswahl von Zitaten in voller Länge wiedergegeben. Zur portugiesischen Sportpresse im Allgemeinen, siehe: PINHEIRO. História da Imprensa desportiva em Portugal.

7 Dietmar Hüser unterscheidet idealtypisch zwischen innerer und äußerer Politisierung des Sportes. Während beispielsweise António Ferros ideologisch aufgeladene Interpretation der halbwegs erfolgreichen portugiesischen Fußballmannschaft bei den olympischen Spielen 1928 zu den Fällen äußerer Politisierung gezählt werden kann, zeichnet sich die innere Politisierung unter anderem dadurch aus, „dass der Sport und seine Verbände auf sämtlichen Ebenen eingebunden sind und aufs Engste verknüpft sind mit den politischen Institutionen des „Politikfeldes Sport“, mit den dort ablaufenden Entscheidungsprozessen sowie den dort vorgenommenen Inhalts- und Zieldefinitionen." Vgl. HÜSER. Moderner Sport und Geschichte als Wissenschaft. Zur politischen, gesellschaftlichen und wirtschaftlichen Verflechtung eines massenkulturellen Phänomens seit der zweiten Hälfte des 19. Jahrhunderts, S. 237. Zu Ferros Berichterstattung von den Olympischen Spielen 1928, vgl. KUMAR. A pureza perdida do desporto. S. 69-76.
} 
1932 Sportspiele noch als Antithese der gesamten Erziehung bezeichnet, die nicht der Veranlagung der Portugiesen entsprächen, ${ }^{8}$ hieß es nun elf Jahre später im Vorwort des Dekretes 32946, dass den Staat an erster Stelle das Problem der körperlichen Erziehung des portugiesischen Volkes interessiere. ${ }^{9}$ Von den Vereinen wurde erwartet, dass sie nicht die Siege ihrer Mannschaften als wichtigstes Ziel ansehen, sondern vielmehr eine noch zu definierende Sportpolitik $\mathrm{zu}$ verfolgen haben, die sich als wahrhaft national erweise und sich nicht in kleinteiliger Vereinsmeierei verliere. Des Weiteren wurden die Vereine aufgefordert, ruinöse Geschäfte einzustellen, denn schließlich leide die Schönheit des Sportes darunter, wenn er sich in eine Lebensart verwandele. Der Gymnastik sollte der größte Stellenwert eingeräumt werden, wobei denjenigen Vereinen Sanktionen drohten, die dieser Aufforderung nur unzureichend nachkamen. Ebenso wurden die Vereine dazu verpflichtet, die medizinische Betreuung und Versorgung ihrer Sportler und Mitglieder zu gewährleisten, was insbesondere im städtischen Raum zur Folge hatte, dass die Vereine hier eine staatliche Aufgabe übernahmen und zur gesundheitlichen Versorgung der Bevölkerung beitrugen.

Diesem Vorwort folgen fünf Kapitel mit insgesamt 97 Paragrafen, in denen eine ganze Reihe von Verboten diese Leitlinien konkretisieren. So wurde den Inspektoren der DGD ein nahezu unbegrenztes Recht zugestanden, in die Verbands- und Vereinspolitik regulierend eingreifen zu können. Das Dekret liest sich nicht nur als eine klare Absage an den Professionalismus, es erschwerte den Vereinen ebenso den Aufbau eigener Jugendmannschaften, da dies in den Zuständigkeitsbereich der MP fiel. Vereinswechsel von Sportlern oder die Beschäftigung von ausländischen Funktionären bedurften ebenso der Zustimmung der DGD, was die sportliche Entwicklung der Vereine bremste und folglich immer wieder durch die kritische Berichterstattung der Vereinspresse angeprangert wurde.

Trotz des klar erkennbaren staatlichen Anspruches, den Verbandssport vollends kontrollieren zu wollen, gestand das Dekret den Vereinen auch gewisse Mitspracherechte zu. An die sportlichen Vertreter erging der Auftrag, Ideen und Vorschläge zu äußern, wie die körperliche Ertüchtigung der Bevölkerung zu

\footnotetext{
${ }^{8}$ ROSÁRIO. O Desporto em Portugal, S. 32.

${ }^{9}$ Die folgenden Passagen sind alle aus dem Dekret 32946 entnommen.
} 
verbessern wäre. Denn laut dem Dekret sei es nicht beabsichtigt, die existierenden sportlichen Strukturen zu zerschlagen. Es gehe vielmehr darum, das Wirken der Vereine mit dem nationalen Interesse in Einklang zu bringen.

Allerdings erwiesen sich manche Bestimmungen des Dekretes auch als unrealistisch, wobei vor allem der Passus zu erwähnen ist, dass bereits 1946 alle Spiele der beiden obersten Fußballligen auf Rasenplätzen stattzufinden haben, was aber erst in den siebziger Jahren erreicht worden ist. ${ }^{10}$ Da diese Bestimmung in den Verantwortungsbereich der Vereine fiel, verwundert es nicht, dass diese eigene Stadionprojekte in der sogenannten „Betonphase“ des portugiesischen Fußballs vorantrieben und viele Vereine in Eigenregie bei überschaubarer staatlicher Subventionierung moderne Stadien errichteten. ${ }^{11}$

Auch auf weiteren Ebenen waren die Vereine sehr erfolgreich, sich von der staatlichen Kontrolle immer weiter zu emanzipieren. So gelang es der Federação Portuguesa de Football (FPF) 1951 den Verbandspräsidenten wieder in Eigenregie zu wählen, während dieser zuvor noch von der DGD ernannt worden war. Ebenso wurden die Bestimmungen zum Aufbau von Jugendmannschaften nach und nach den Wünschen der Vereine angepasst, denen es auch gelang, den seit den zwanziger Jahren existierenden Scheinamateurismus in einen offiziell anerkannten Professionalismus zu überführen und die staatlichen Eingriffe bei Spielertransfers zu minimieren. ${ }^{12}$ Dieser Prozess der Emanzipierung konnte bis Mitte der fünfziger Jahre abgeschlossen werden und lässt sich in den kontroversen Debatten der Sportpresse verfolgen.

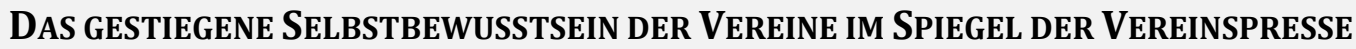

Dass Vereinszeitungen immer wieder die Wichtigkeit ihrer Vereine im gesellschaftlichen Bereich betonen, ist nachvollziehbar und bereits vor der staatlichen Vereinnahmung des Vereinssportes zu erkennen. Schon im Jahre 1936 wies der Herausgeber des „Boletim do Sporting Clube de Portugal“, Dr. José Salazar

\footnotetext{
${ }^{10}$ SERRADO. História do Futebol Portugûes. Das origens ao 25 de abril. S. 439.

${ }^{11}$ PINHEIRO. Futebol e Política na Ditadura: Factos e Mitos. S. 67.

12 KUMAR, A pureza perdida do desporto. Futebol no Estado Novo. S. 210-231.
} 
Carreira, ${ }^{13}$ darauf hin, dass der Sport es schaffe, Angehörige verschiedener sozialer Klassen in Kameradschaft, gegenseitigem Respekt und beiderseitigem Nutzen zu vereinen. ${ }^{14}$ Dass Salazar Carreira dies mit einer klaren Absage an den Professionalismus verband, überrascht nicht, zumal er in den folgenden Jahren Karriere als Inspektor der DGD machte und stets versuchte, die erzieherische Funktion des Sportes in den Vordergrund $\mathrm{zu}$ stellen und von negativen Begleiterscheinungen des Massenspektakels abzutrennen. In den vierziger und fünfziger Jahren sind aber in den Vereinszeitungen auch andere Interpretationen zu erkennen, die zeigen, wie die immer selbstbewusster auftretenden Vereine die ideologischen Vorgaben mit ihren Vereinsinteressen verbanden.

So bezog die Zeitung des Vereines „Os Belenenses“ im Dezember 1943 explizit Stellung zum Dekret 32946. Sie sah darin keine Bedrohung der Vereine, sondern vielmehr eine sinnvolle Maßnahme des Staates, da der private Charakter der Vereine anerkannt worden sei. Dies entspreche voll und ganz dem politischen und sozialen Aufbau der portugiesischen Nation, in der kein Platz für totalitäre Tendenzen sei und private Initiativen stets das Allgemeinwohl im Blick haben. ${ }^{15}$ Da zu diesem Zeitpunkt die Kriegsniederlage der als totalitär geltenden Achsenmächte bereits abzusehen war, schadete dieser dezente Hinweis auf die Andersartigkeit der portugiesischen Diktatur sicherlich nicht, zumal damit auch ablehnende Äußerungen Salazars zum Totalitarismus aufgegriffen wurden ${ }^{16}$ und größere Gestaltungsmöglichkeiten der Vereine mit der salazaristischen Ideologie in Einklang gebracht werden konnten.

\footnotetext{
${ }^{13}$ Als ehemaliger Athlet, Arzt und Autor hatte er viele hohe Funktionen im portugiesischen Sport inne: Präsident von Sporting 1925/26, Präsident der FPF 1930/31 sowie Inspektor der DGD von 1943 bis 1964.

${ }^{14}$ CARREIRA SALAZAR. A elevada missão dos clubs e os objectivos a que deve atender, S. 1. Im Original: Formadas pelo aglomerado de indivíduos das classes mais diversas, estabelecemse por seu intermédio laços de camaradagem entre pessoas que a vida destinara a viver separadas, e os princípios sagrados da solidariedade, do auxílio mútuo, do respeito pessoal, desenvolvem-se no contacto desportivo com evidentes vantagens mútuas: os de cima lucram em ser melhor apreciados, os de baixo progridem no convívio com criaturas de educação e cultura superior à sua.

${ }^{15}$ C.F. Os Clubes e a educação Física, S. 1. Im Original: A orgânica política e social da Nação Portuguesa assenta nêste princípio, evitando prudentemente uma planificação totalitária onde o fim e interêsse geral do sistema domina por perfeito absoluto e actividade privada dos indivíduos e grupos.

${ }^{16}$ Etwa in einer Rede am 6. Juli 1937. Vgl. SAMPAIO. Salazar. Pensamento e Doutrina Política, S. 139.
} 
Dass Vereins- und Staatsinteressen sich bedingen, wollte der Verein durch weitere Anstrengungen beweisen. Dies betreffe nicht nur den Bau neuer Sportstätten, sondern auch die Aufnahme von Sportlern und Vereinsmitgliedern aus unteren sozialen Klassen in die hierarchisch aufgebauten Strukturen des Vereines. In diesem Zusammenhang wurde argumentiert, dass die Vereine als staatlich legitimierte Akteure die wachsende Stadtbevölkerung disziplinieren und in die Gesellschaft integrieren können. Natürlich sei deswegen eine genauere Abstimmung des Staates mit den Vereinen wünschenswert, zumal es in der Vergangenheit auch immer wieder Probleme mit der Disziplin rund um die Sportstätten gegeben habe. Laut dem Autor sind aus all diesen aufgezählten Gründen Vereinsinteressen und Gemeinwohl nicht als Antagonismen aufzufassen, sondern vielmehr kann letzteres durch ersteres erreicht werden. Dieses Motiv der Komptabilität findet sich auch immer wieder in der Vereinszeitung „O Benfica“, die im Jahre 1954 sogar Ministerpräsidenten Salazar direkt zitierte, um die positive Rolle des Sportes hervorzuheben. ${ }^{17}$ Der Sprachstil dieser Artikel ähnelt hierbei oft den ideologisch aufgeladenen und wenig konkreten Passagen des Dekretes 32946, doch finden sich auch immer wieder Belege, die das Zusammenspiel von staatlichen und Vereinsinteressen deutlich veranschaulichen. Als ein Beispiel unter vielen dient hier Sporting Clube de Portugal, das im Jahre 1947 seinen neuen Vereinssitz mit integriertem Kino und medizinischer Abteilung für seine 15000 Mitglieder einweihte. $\mathrm{Zu}$ diesem Anlass hielt der spätere portugiesische Ministerpräsident Adelino da Palma Carlos in der Vereinszeitung fest, dass der Staat das Gebäude dem Verein im Tausch gegen das alte Vereinsgelände überlassen habe, damit Sporting einerseits seinen wichtigen Dienst an der Nation nun noch vollkommener ausführen könne und andererseits der Bitte des Staates entsprochen worden sei, Zugriff auf den alten Vereinssitz zu haben. ${ }^{18}$

Ein weiteres Beispiel für konkretes soziales Engagement der Vereine liefert „Os Belenenses“ aus dem Jahre 1943, die der Eröffnung einer Bibliothek durch den benachbarten Verein Atlético Clube de Portugal aus Alcântara als Beweis der

\footnotetext{
${ }_{17}^{17}$ PIRES. O Elogio do Desporto, S. 3.

${ }^{18}$ PALMA CARLOS. Alocução proferida na sessão solene de inauguração da Séde, S. 1.
} 
wichtigen Rolle der Vereine im Bereich von Erziehung und Kultur ansah und dementsprechend würdigte. ${ }^{19}$

Dass solche Maßnahmen die staatliche Wertschätzung der Vereine steigern konnten, konstatierte Alberto Freitas vom Atlético Clube im Jahre 1944, der aber auch festhielt, dass die Vereinsarbeit immer noch durch Verleumdungen, Eifersüchteleien sowie Intrigen, denen der Sport überdrüssig sei, bedroht werde und dieser Reflex einer geistigen Inferiorität nur dann verschwinden könne, wenn sich die allgemeine Erziehung verbessere. ${ }^{20}$

Dass der Verein Altético ${ }^{21}$ aber kaum Verbesserungen im Bereich der Erziehung feststellte, wurde im Jahre 1946 deutlich, als das Vorstandsmitglied Monte-Castro $\mathrm{zu}$ dem drastischen Schluss kam, dass es in Portugal trotz anderslautender Stellungnahmen von Premierminister Salazar überhaupt keine körperliche Erziehung gebe, denn sonst wäre die Kindersterblichkeitsrate nicht so hoch und statt Verbesserungen könne man nur eine stündlich wachsende Enttäuschung der Vereinsanhänger registrieren. ${ }^{22}$

Diese harsche Kritik, die erstaunlicherweise die Pressezensur unbeschadet überstand, reflektiert das gestiegene Selbstbewusstsein der Vereine, die es nicht scheuten, den Staat immer wieder aufs Schärfste zu kritisieren oder sein Engagement als unzureichend kennzeichneten, was sich am Beispiel des Estádio da Tapadinha von Atlético Clube de Portugal zeigt. Auf die Frage, ob dieses Stadion ein Werk des Staates sei, gab der Präsident des Vereins, Joaquim Nobre, unmissverständlich $\mathrm{zu}$ Protokoll, dass das Stadion nur aufgrund des aufopferungsvollen Wirkens der Vereinsmitglieder gebaut werden konnte, es damit ein Werk des Vereines sei und sich die Zuwendungen des Staates in einem beschaulichen Rahmen hielten. ${ }^{23}$

\footnotetext{
${ }^{19}$ U.A. Atlético Clube de Portugal, S. 10.

${ }^{20}$ FREITAS. Como eu vejo o Atlético, 1944, S. 5. Im Original: [O] Atlético [...] sentiu à sua volta o ambiente de apreço a que tem direito quem trabalha com boas intenções e indiferente às maledicências, às intrigas, às invejazinhas, de que o meio desportivo português é farto, e que não são mais que o reflexo duma inferioridade de espírito que só desaparecerá quando a educação geral fôr mais profunda.

${ }^{21}$ Zur Geschichte Atléticos in den vierziger und fünfziger Jahren, Vgl. WEIBMANN. Atlético, capital dum país que se chama Alcântara, S. 319-344.

${ }_{22}$ MONTE-CASTRO. No Momento Presente... política, não! união, sim!, S. 2,25.

${ }^{23}$ MONTE-CASTRO. Os <<Atléticos de Portugal >> falam alto do seu orgulho e dos seus designos pela boca prestigiosa do seu novo presidente, S. 7,11.
} 
Trotz dieser Kritik, die nicht nur von Atlético geäußert worden ist, bemühten sich aber alle Vereine ebenso, die in ihren Augen selbstverständlich ruhmreiche eigene Vereinsgeschichte in einen nationalen Kontext einzubetten. Diese Vorgehensweise kann zum einen als weiteren Beleg für das wachsende Selbstbewusstsein der Vereine gelten, da sich die Vereine nun selber als stolze Produkte portugiesischer Größe interpretierten. Zum anderen ist diese Vorgehensweise aber auch als eine Art schmeichelnde Unterwerfung unter die nationalistische Konzeption des Estado Novo aufzufassen. So war sich beispielsweise Sporting sicher, dass der Verein durch die Errichtung seiner zahlreichen Filialen auf dem ganzen portugiesischen Staatsgebiet dem Wohle des portugiesischen Volkes diene. ${ }^{24}$ In „O Benfica“ wurde behauptet, dass der Verein in der Seele des Volkes wohne und das Volk die Seele von Benfica sei, da es keinen Flecken portugiesischer Erde gebe, in welchem Benfica nicht existiere. ${ }^{25}$ Auch Atlético sah den Verein fest in portugiesischer Erde verwurzelt, zumal man auch vom Stadion auf den ruhmreichen Tejo blicken könne, von wo aus alle Größe Portugals ihren Ursprung genommen habe. ${ }^{26}$ In Belém betonte man bei der Eröffnung des Stadions, dass von diesem Stadtteil die Entdeckungsfahrten unter göttlicher Führung ihren Anfang nahmen und die Vereinsfarbe blau mit der Farbe des Himmels und des Meeres korrespondiere. ${ }^{27}$

Die positiven Effekte einer solchen Berichterstattung für das diktatorische Regime sind bereits an anderer Stelle bezüglich der propagandistischen Eröffnungsfeiern großer Stadien sowie der Erfolge der portugiesischen

${ }^{24}$ GAMA TAVARES. Iniciativa triunfante, S. 2. Im Original: O sportinguismo nasceu verdadeiramente há trinta e tantos anos, quando o prestigio do Sporting começou a fecundar a criação de pequenos clubes na provincia, os muitos Sportings que pontilham os mapas do Território Português. E desde essa época a heráldica do leão branco em campo verde inspirou e guiou, na sua projecção, a maior massa de vontades, de dedicações e de realizações que têm ilustrado o desporto da Nossa Terra.Não sentimos nós hoje, os sportinguistas, menos sólida a nossa fé nos destinos do Sporting e do Sportinguismo nem menor o orgulho pela obra que tem levado a cabo em beneficio da Raça Portuguesa.

${ }^{25}$ U.A. O Povo! O Benfica!, S. 4. Im Original: O Benfica vive na alma do povo, o povo é a alma do Benfica. Onde está um, está o outro. E asim não há cidade, nem vila, nem aldeia de Portugal onde não pulse, vibre e flameje a alma sã do povo, do mesmo modo nenhuma terra portuguesa existe onde o Benfica não existe.

${ }^{26}$ PAIVA. O Estádio da Tapadinha, S. 9.

${ }^{27}$ U.A. A saudação do Beleneneses , S. 6. 
Nationalmannschaft in den sechziger Jahren hinreichend analysiert worden. ${ }^{28} \mathrm{Im}$ Folgenden wird daher der Fokus auf eine andere Problematik gelegt. Inwiefern nutzten die Vereine ihren Beitrag zur körperlichen Ertüchtigung des Volkes aus, um vom Staat weiteres Entgegenkommen für Vereinsbelange einzufordern?

\section{ERZIEHUNG UND SPEKTAKEL: ZWEI SEITEN EINER MEDAILle?}

In der portugiesischen Nationalversammlung wurde 1949 mit Wohlwollen festgestellt, dass die Steuereinnahmen aus Fußballspielen von 1942 bis 1948 um das Sechsfache anstiegen und 1948 bereits dreimal mehr Einnahmen als das Kino generierten. Obwohl die erzieherische Mission der Vereine in den vierziger Jahren gesetzlich anerkannt worden war und der Staat auch staatliche Subventionen zur Errichtung neuer Stadien gewährte, stellte diese rigorose Besteuerungspraxis die Vereine vor existentielle Probleme. Bereits im Jahre 1947 wurde die Besteuerung erstmalig reformiert, wobei die steuerlichen Abgaben an die Stadionkapazität gekoppelt wurden. Eigentlich als Steuererleichterung für die Vereine gedacht hatte dieses neue Abgabensystem jedoch zur Folge, dass in vielen Fällen die zu bezahlenden Steuern den Reingewinn aus den Zuschauereinnahmen überstiegen. Nach heftigen Protesten der Vereine wurde die Besteuerung 1951 abermals reformiert und dem im Estádio Nacional praktizierten Abgabensystem angepasst. ${ }^{29}$ Die Vereinszeitungen begleiteten diesen Prozess mit scharfer Kritik, wobei sie das Motiv des staatlichen Erziehungsauftrages der Vereine immer vehementer mit der Forderung nach einem gerechteren Besteuerungssystem verbanden.

Während 1946 „Os Belenenses“ die Tatsache, dass beim Heimspiel gegen C.U.F die Aus- und staatlichen Abgaben die Einnahmen um mehr als das Doppelte überstiegen, noch fassungslos zur Kenntnis nahm und ohne konkret zu werden Verbesserungen für diese beklemmende Situation einforderte, ${ }^{30}$ warf die gleiche Zeitung bereits einige Monate später die Frage auf, wie kleinere Vereine auf dem

${ }^{28}$ Etwa ACKERMANN. Fußball und nationale Identität in Diktaturen. Spanien, Portugal, Brasilien und Argentinien oder COELHO. Portugal. A Equipa de todos nós.

${ }^{29}$ Zu diesem ganzen Abschnitt vgl. WEIBMANN. Fußball in der portugiesischen Diktatur, S. 25-34.

${ }^{30}$ U.A. Para 8 contos de receita uma despesa de 16 contos, S. 2. 
Land mit dieser Steuerlast umgehen, die sie umso härter treffe, da sie dies nicht wie mancher Großverein durch viele Mitgliedsbeiträge auffangen können. ${ }^{31}$ Doch mit Pitta Castelejo schlug auch ein Vertreter eines großen Vereins wie Sporting bereits 1946 angesichts der hohen Abgaben Alarm. In seiner Rubrik „Retalhos“ verlangte er eine spürbare Steuererleichterung, da sonst bald der Tag bald käme, an dem sich die Vereine gezwungen sähen, jedwede Verantwortung abzugeben und dies zur Paralyse des gesamten portugiesischen Sportes führe. ${ }^{32}$ Auch wenn in solchen Artikeln immer wieder das Stilmittel der Übertreibung genutzt und die eigene Situation schlimmer dargestellt wurde, als sie wirklich war, findet sich in diesem Artikel eine Frage an den Staat, die auch in den Publikationen anderer Vereine Teil der Argumentation für niedrigere Steuern wurde. Können die Vereine in einer solch angespannten finanziellen Situation ihr Ziel verwirklichen, das darin bestehe, das Individuum durch körperliche Ertüchtigung zu disziplinieren, damit es zu einem nützlichen Mitglied der Gesellschaft werden könne? ${ }^{33}$

Diese Frage stellte beispielsweise auch Raul Jorge im „Boletim do Atlético“, der die Bilanz der Einnahmen aus allen Heimspielen von Atlético präsentierte und dabei festhielt, dass über 60\% von Aus- und Abgaben absorbiert wurden. Für einen Sportverein, der nicht als kommerzielles oder industrielles Unternehmen betrachtet werden könne, sei dies eine zu hohe Last, zumal die Vereine durch ihr Sportangebot eine nützliche soziale Funktion übernehmen würden und dem Staat damit einen großen Dienst erweisen. ${ }^{34}$ Auch „O Benfica“ stimmte in dieses allgemeine Klagelied ein und merkte an, dass der Fußball keine Industrie sei. Das Abgabensystem von 1947 sei ungerecht gegenüber dem Sport, der für den Autor unverständlicherweise höher besteuert werde als der Stierkampf, die Kinos oder das Theater. ${ }^{35}$

\footnotetext{
${ }^{31}$ U.A. Dois problemas importantes para o desporto, S. 2.

${ }^{32}$ CASTELEJO. Retalhos, S. 8. Im Original: [D]aqui levantamos o nosso grito para o juntar aos outros, clamando com sincera veemência e num arroubo de verdade sentida. - que é necessário, muito necessário, rever o actual sistema dos Impostos que oneram as organizações desportivas, porque a continuar de pé a sua vigência, não vira longe dia em que os Clubes se veiam forçados a declinar toa e qualquer responsabilidade, na paralização desportiva dos atletas portugueses.

${ }^{33}$ CASTELEJO. Retalhos, S. 8. Im Original: Podem os clubes, a manter-se esta angustiosa situação, cumprir com o fim para que se propuzeram, - a valorização do indivíduo, perante a sociedade, pela prática metódica, proficiente e disciplinada, dos excercícios físicos, S. 8.

${ }_{34}$ JORGE. Receitas e despesas, S. 9.

${ }^{35}$ U.A. Uma situação injusta para o Desporto, S. 1/2.
} 
Auch wenn die Vereine natürlich versuchten, ihre Einnahmen dadurch zu vergrößern, dass sie immer wieder neue Kampagnen zur Mitgliedergewinnung starteten, hielt Armando Matos für den Fall Atlético fest, dass dies nur Sinn mache, wenn der Staat gleichzeitig Verständnis dafür aufbringe, dass das derzeitige Abgabensystem die Vereine in den Ruin treibe. ${ }^{36}$

Selbst wenn an dieser Stelle nicht zu klären ist, inwiefern sich der Staat von dieser Berichterstattung beeinflussen ließ, so ist dennoch festzuhalten, dass die Vereine die Vorgabe des Staates, dass die körperliche Erziehung eine Wichtigkeit besäße, nun auch als rhetorische Waffe gegen staatliche Restriktionen nutzen konnten. Ähnliches ist zu konstatieren, wenn die Vereine an anderen Verboten wie dem Professionalismus rüttelten und diesen als Lösungsvorschlag für die angespannte finanzielle Situation ins Gespräch brachten ${ }^{37}$ oder die hohen Transportkosten innerhalb des Landes kritisierten und hier spezielle Tarife für die vielreisenden Vereine einforderten. ${ }^{38}$

\section{SCHLUSS}

Betrachtet man die Situation des portugiesischen Sportes zu Beginn der sechziger Jahre, als vor allem der Fußball in ein goldenes Zeitalter eintreten konnte, war von den restriktiven Bestimmungen des Dekretes 32946 aus dem Jahre 1943 nicht mehr viel übrig. Die Vereine emanzipierten sich in den vierziger und fünfziger Jahren immer mehr von der staatlichen Kontrolle, die sie zunehmend auch durch kritische Beiträge in ihren Vereinsorganen herausforderten. Es gelang ihnen hierbei, staatliche Vorgaben wie ihr Beitrag zur körperlichen Ertüchtigung des Volkes für die Entwicklung der Vereine nutzbar zu machen und als Argument für Steuererleichterungen oder die Einführung des Professionalismus umzukehren. Darüber hinaus wuchs das Selbstvertrauen der Vereine, da sie überwiegend in Eigenregie ihre Stadien errichteten und sich ihrer gesellschaftlichen Macht bewusst waren, die parallel zu ihren Mitgliederzahlen und Sympathisanten stetig

\footnotetext{
${ }^{36}$ MATOS. mais gente menos encargos, S. 6 .

${ }^{37}$ BAPTISTA. Amadorismo e Profissionalismo, S. 3.

${ }^{38}$ U.A. Um problema importante. Os clubes e os transportes, S. 3.
} 
anstieg. Es steht außer Frage, dass dieser Aufstieg nicht nur durch Konfrontation mit dem Staat erreicht worden oder geradlinig verlaufen ist. Der Staat sanktionierte weiterhin die Vereine im Falle von Zuschauerausschreitungen sehr hart und fürchtete ebenso, dass der Zusammenhalt der Nation dadurch gefährdet wäre, wenn sich die Bevölkerung in unversöhnlich gegenüberstehende Anhänger unterschiedlicher Vereine aufspalten würde. Die ambivalente Beziehung des Sportes zur Diktatur wird an dieser Stelle besonders deutlich. Trotz dieser negativen Begleiterscheinungen rund um den Vereinssport profitierte der Staat dadurch, dass die Vereine zunehmend in ihrer Berichterstattung oder dem Schreiben ihrer eigenen Geschichte immer wieder auf nationalistische Narrative zurückgriffen.

Dass sich die sportlichen Massenveranstaltungen hoch besteuern ließen, war ein weiterer Vorteil für die Diktatur. Zwar konnten die Vereine mit ihren Protesten Steuererleichterungen erreichen, doch bleibt festzuhalten, dass der Emanzipierung der Vereine Grenzen gesetzt waren und sie trotz aller Kritik weiterhin auf das Wohlwollen des Staates angewiesen waren. In dieser Perspektive wären weitere Studien wünschenswert, wie die Vereine mit ihrer ambivalenten Situation in der Diktatur umgingen. Vor allem bei den städtischen Großvereinen wären hier spannende Ergebnisse zu erwarten, da sich der gesellschaftliche Wandel im Zuge der verspäteten Industrialisierung Portugals und der damit verbundenen zunehmenden Verstädterung in der Entwicklung der Vereine widerspiegelte. Die daraus resultierenden sozialen Probleme stellten die Diktatur vor neue Herausforderungen, bei denen die Vereine gleichzeitig die Rolle eines staatlichen Verbündeten in der Disziplinierung der Massen, aber auch die eines Gegners einnehmen konnten, wenn sich die Vereinsanhänger zunehmend von der Diktatur abwenden. Bei der Annäherung an diese Problematiken würden sich die Vereinszeitungen als vielversprechende Fundgrube erweisen und mit einer alten Annahme von Tom Gallagher aufräumen, dass die portugiesische Presse während der Diktatur vermutlich die langweiligste der Welt gewesen sei. ${ }^{39}$

\footnotetext{
${ }^{39}$ GALLAGHER. Portugal. A twentieth-century interpretation, S. 101. Zitiert nach: ABELE. Kein kleines Land, S. 34.
} 


\section{LITERATURVERZEICHNIS}

ABELE, Christiane. Kein kleines Land. Die Kolonialfrage in Portugal 1961 1974. Göttingen: Wallstein, 2017.

ACKERMANN, Jürg. Fußball und nationale Identität in Diktaturen. Spanien, Portugal, Brasilien und Argentinien. Zürich: Lit-Verlag, 2013.

BAPTISTA. Amadorismo e Profissionalismo 03.03.1947 Boletim do Sporting Club de Portugal, Lisboa 3 mar. 1947. S. 3.

CARREIRA SALAZAR. A elevada missão dos clubs e os objectivos a que deve atender, 08/10.36. Boletim do Sporting Club de Portugal, Lisboa ago.-out. 1936. S. 1.

CASTELEJO. Retalhos 30.10.1946. Boletim do Sporting Club de Portugal, Lisboa 30 out. 1946 . S. 8.

C. F. Os Clubes e a Educação Física 12.1943, Os Belenenses, Lisboa dez. 1943. S. 1.

COELHO, João Nuno. Portugal. A Equipa de todos nós. Nacionalismo, Futebol e Media. Porto: Afrontamento, 2001.

FREITAS. Como eu vejo o Atlético 1944, Número único comemorativo do ano em que o A.C.P. se afirma como grande colectividade desportiva nacional, Lisboa 1944. S. 5.

GALLAGHER, Tom. Portugal. A twentieth-century interpretation. Manchester: Univ. Press, 1983.

GAMA TAVARES. Iniciativa triunfante 15.11.1948, Boletim do Sporting Club de Portugal, Lisboa 15 nov. 1948. S. 2.

HÜSER, Dietmar. Moderner Sport und Geschichte als Wissenschaft. Zur politischen, gesellschaftlichen und wirtschaftlichen Verflechtung eines massenkulturellen Phänomens seit der zweiten Hälfte des 19. Jahrhunderts. Neue politische Literatur, v. 51, n. 2/3, S. 223-264, 2006.

JORGE. Receitas e despesas, 02.1950, Boletim do Atlético, Lisboa fev. 1950. S. 9.

KUMAR, Rahul. A pureza perdida do desporto. Futebol no Estado Novo. Lisboa: Edições Paquiderme, 2017.

MATOS. mais gente menos encargos 08.1950, Boletim do Atlético, Lisboa aug. 1950. S. 6.

MONTE-CASTRO. No Momento Presente...política, não! união, sim! 1946. Atlético Clube de Portugal. Número especial comemorativo do seu IV aniversário, Lisboa 1946. S. 2,25. 
MONTE-CASTRO. Os <<Atléticos de Portugal >> falam alto do seu orgulho e dos seus designos pela boca prestigiosa do seu novo presidente 1946. Atlético Clube de Portugal. Número especial comemorativo do seu IV aniversário, Lisboa 1946. S. 5,7,11.

MELO, Daniel. A cultura popular no Estado Novo. Coimbra: Angelus Novus, 2010. MINISTÉRIO DA EDUCAÇÃO NACIONAL. Decreto-lei n. ${ }^{\circ} 32: 241$. Diário do Gôverno, I série-Número 208, 5 set. 1942. S. 1135-1139.

MINISTÉRIO DA EDUCAÇÃO NACIONAL. Decreto-lei n. ${ }^{\circ} 32: 946$. Diário do Gôverno, I série-número 162, 3 ago. 1943. S. 491-499.

PAIVA. O Estádio da Tapadinha 12.1949, Boletim do Atlético, Lisboa dez. 1949. S. 9.

PALMA CARLOS. Alocução proferida na sessão solene de inauguração da Séde 10.07.1947, Boletim do Sporting Club de Portugal, Lisboa 10 jul. 1947. S. 1.

PINHEIRO, Francisco. Futebol e Política na Ditadura: Factos e Mitos. In: DOMINGOS, Nuno; TIESLER, Nina Clara (Ed.): Futebol português - política, género \& movimento. (História e desporto). Porto: Afrontamento, 2012. S. 47-82.

PINHEIRO, Francisco. História da Imprensa desportiva em Portugal. Porto: Edições Afrontamento, 2010.

PIRES. O Elogio do Desporto 21.07.1953, O Benfica, Lisboa 21 jul. 1953. S. 3. ROSÁRIO, Alberto. O Desporto em Portugal, Lisboa: Edição Instituto Piaget, 1996.

ROSAS, Fernando. O salazarismo e o homem novo: ensaio sobre o Estado Novo e a questão do totalitarismo. Análise Social, v. 157, S. 1031-1054, 2001.

SAMPAIO, Henrique. Salazar - pensamento e doutrina política, Lisboa: Verbo, 2007.

SERRADO, Ricardo. História do Futebol Português - das origens ao 25 de abril, v. 1, Lisboa: Edição Rui Pedro Braz, 2010.

U. A. A saudação do Beleneneses 04.09.1955. A Bola, Lisboa 4 set. 1956. S. 6.

U. A. Atlético Clube de Portugal 03.1953, Os Belenenses, Lisboa mar. 1943. S. 10.

U. A. Dois problemas importantes para o desporto 02.1947, Os Belenenses, Lisboa fev. 1947. S. 2.

U. A. O Povo! O Benfica! 28.04.1954, O Benfica, Lisboa 28 abr. 1954. S. 4.

U. A. Para 8 contos de receita uma despesa de 16 contos 09.1946, Os Belenenses, Lisboa set. 1946. S. 2.

U. A. Uma situação injusta para o Desporto 19.07.1947, Sport Lisboa e Benfica, Lisboa 19 jul. 1947. S. 1/2.

U. A. Um problema importante. Os clubes e os transportes 26.04.1947, Sport Lisboa e Benfica, Lisboa 26 abr. 1947. S. 3. 
WEIBMANN, Thomas. Atlético, capital dum país que se chama Alcântara. In: PINHEIRO, Teresa; SARTINGEN, Kathrin (Ed.). Alles andere als unsichtbar / Tudo menos invisível. Theater, Literatur und Film der lberoromania zwischen Kunst und Leben / Teatro, literatura e cinema no mundo ibero-românico entre vida e arte. Frankfurt am Main: Peter Lang, 2017, S. 319-344.

WEIBMANN, Thomas. Fußball in der portugiesischen Diktatur. Unveröffentlichte Masterarbeit im Fachbereich 05 Geschichtswissenschaften Universität Kassel, Kassel 2010.

Recebido para publicação em: 07 set. 2017. Aprovado em: 20 dez. 2017. 